\title{
A COMPARATIVE STUDY BETWEEN FREQUENCY-MODULATED CONTINUOUS WAVE LADAR AND LINEAR MODE LIDAR
}

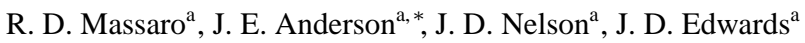

${ }^{\text {a }}$ Remote Sensing and Fluorescence Spectroscopy Lab, US Army Corps of Engineers ERDC,

7701 Telegraph Road Alexandria, Virginia 22315 - John.Anderson@ usace.army.mil

\author{
Commission VI, WG VI/4
}

KEY WORDS: LiDAR, LADAR, range resolution, laser, terrestrial scanning, frequency-modulated, continuous wave

\begin{abstract}
:
Topographic Light Detection and Ranging (LiDAR) technology has advanced greatly in the past decade. Pulse repetition rates of terrestrial and airborne systems have multiplied thus vastly increasing data acquisition rates. Geiger-mode and FLASH LiDAR have also become far more mature technologies. However, a new and relatively unknown technology is maturing rapidly: Frequency-Modulated Continuous Wave Laser Detection and Ranging (FMCW-LADAR). Possessing attributes more akin to modern radar systems, FMCWLADAR has the ability to more finely resolve objects separated by very small ranges. For tactical military applications (as described here), this can be a real advantage over single frequency, direct-detect systems. In fact, FMCW-LADAR can range resolve objects at $10^{-7}$ to $10^{-6}$ meter scales. FMCW-LADAR can also detect objects at greater range with less power. In this study, a FMCWLADAR instrument and traditional LiDAR instrument are compared. The co-located terrestrial scanning instruments were set up to perform simultaneous 3-D measurements of the given scene. Several targets were placed in the scene to expose the difference in the range resolution capabilities of the two instruments. The scans were performed at or nearly the same horizontal and vertical angular resolutions. It is demonstrated that the FMCW-LADAR surpasses the perfomance of the linear mode LiDAR scanner in terms of range resolution. Some results showing the maximum range acquisition are discussed but this was not studied in detail as the scanners' laser powers differed by a small amount. Applications and implications of this technology are also discussed.
\end{abstract}

\section{INTRODUCTION}

\subsection{Background}

Pulsed light detection and ranging (LiDAR) systems for terrestrial surveying and topographic mapping have made great strides in the past 10 to 15 years. Although terrestrial laser scanning was first used in the 1960's(Shan and Toth, 2009), only recently have the scanning mechanisms and computer hardware advanced enough to support topographic measurements within a reasonable amount of time. Terrestrial LiDAR instruments are now able to collect millions of accurately georegistered points within several seconds. While pulsed LiDAR technology is certainly more mature than other approaches, frequency-modulated continuous wave (FMCW) laser detection and ranging (LADAR) is rapidly gaining ground due to several key benefits. First and most evident, is the difference in range resolution between the two technologies. Pulsed LiDAR is limited in range resolution by the width of the emitted laser pulse. A typical conventional LiDAR system emits Gaussian-shaped laser pulses. Meanwhile, FMCW-LADAR is limited in range resolution by the chirped bandwidth of the emitted beam(Reibel et al., 2014). An example of a chirped bandwidth from a FMCW-LADAR system can be seen in Reibel et al(Reibel et al., 2010), Figure 2. Secondly, FMCW-LADAR can detect doppler motions in the returned laser pulse. Finally, FMCW-LADAR requires less power to achieve range measurements or, conversely, can detect objects at a greater range than a pulsed LiDAR having the same power. In this study, the range resolutions of a conventional, pulsed LiDAR system and a FMCW-LADAR system are compared and contrasted using real-world targets.

\footnotetext{
${ }^{*}$ Corresponding author.
}

\subsection{Instrument specifications}

The instruments used in this comparison experiment were a Riegl VZ-400 3D terrestrial laser scanner and a Bridger HRS-3D-1W imager. The VZ-400 is a conventional, pulsed Class I scanning laser system with a $360^{\circ}$ horizontal and $100^{\circ}$ vertical field-ofview (FOV). The HRS-3D is a FMCW Class IIIb scanning laser system with a $360^{\circ}$ horizontal and $60^{\circ}$ vertical FOV. The physical dimensions of the scanner heads are very similar (see Table 1). The HRS-3D has a separate processing unit whereas the VZ400 performs its processing within the scanner unit. The HRS-3D weighs about twice as much as the VZ-400 and requires about 3 times as much power. Both scanners are easily tripod mounted.

The VZ-400 and HRS-3D lasers both emit at $1.55 \mu \mathrm{m}$. The maximum pulse repetition rates for the VZ-400 and HRS-3D are 300 $\mathrm{kHz}$ and $48 \mathrm{kHz}$, respectively. The beam divergences for the VZ400 and HRS-3D are $0.35 \mathrm{mrad}$ and $0.1 \mathrm{mrad}$, respectively.

\begin{tabular}{c|cc}
\hline & Riegl VZ-400 & Bridger HRS-3D \\
\hline $\begin{array}{l}\text { Scanner head } \\
\text { (h x dia) }\end{array}$ & $31 \mathrm{~cm} \mathrm{x} \mathrm{18} \mathrm{cm}$ & $28 \mathrm{~cm} \mathrm{x} \mathrm{20} \mathrm{cm}$ \\
\hline Processor size & None & $48 \mathrm{~cm} \mathrm{x} 43 \mathrm{~cm} \mathrm{x} \mathrm{23} \mathrm{cm}$ \\
\hline Weight & $9.6 \mathrm{~kg}$ & $19 \mathrm{~kg}$ \\
\hline Power & $65 \mathrm{~W}$ & $350 \mathrm{~W}$ \\
\hline
\end{tabular}

Table 1: Size, weight, and power comparisons of the two scanners 


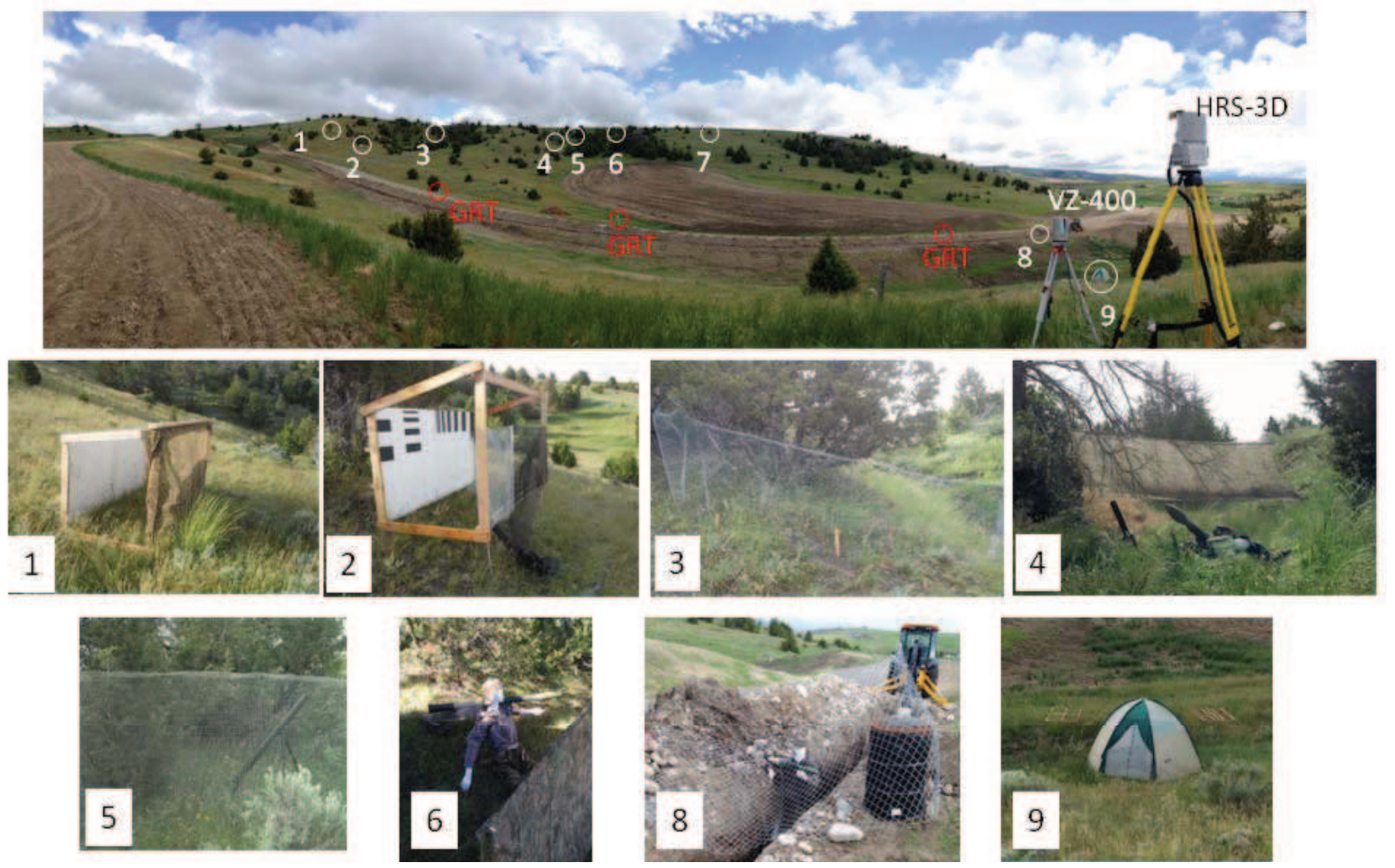

Figure 1: (Top) Overview of scan area and target placements (Middle and bottom rows) Images of targets [No image of target 7 available]

\section{DATA COLLECTION}

For this series of tests, we explored a variety of tactical scenarios and targets of interest to compare and contrast the technologies. A data acquisition site was chosen at approximate coordinates: $45.661^{\circ} \mathrm{N}, 111.345^{\circ} \mathrm{W}$. The site is approximately 14 miles west of Bozeman, MT. A Riegl VZ-400 terrestrial laser scanner and a Bridger Photonics HRS-3D-1W terrestrial laser scanner were mounted on tripods and placed side-by-side (Figure 1, top). Several targets were placed in the scene. Targets 1 through 7 were placed at ranges between approximately 200 and 250 meters. Targets 8 and 9 were placed at $68 \mathrm{~m}$ and $45 \mathrm{~m}$, respectively. The targets were designed to simulate sniper blinds and to test the range ambiguity issues that typically arise in direct detect systems. The targets also were designed to evaluate the obscurant penetration ability of the scanners. Camouflage netting and various types of fencing were used to obscure targets. A list of the targets, their descriptions, and their ranges from the scanners are shown in Table 2 .

The scans from both instruments were performed with the measurement angular resolution set at $0.001^{\circ} \times 0.001^{\circ}$. The exception was for Target 9 which was scanned at a $0.005^{\circ} \times 0.005^{\circ}$ resolution. The target scans were not made simultaneously to avoid interference introduced by each of the system's lasers.

\section{COMPARISONS}

The range ambiguity and obscurant penetration performance of the two scanner technologies was explored. A test of the maximum range of the scanners was also made. The comparisons between the scanners on these topics are discussed in the following sections.

\begin{tabular}{|c|c|c|}
\hline Target \# & Range(m) & Description \\
\hline 1 & 220 & $\begin{array}{l}\text { Sniper blind with camou- } \\
\text { flage netting }\end{array}$ \\
\hline 2 & 185 & $\begin{array}{l}\text { Sniper blind with calibra- } \\
\text { tion pattern }\end{array}$ \\
\hline 3 & 249 & $\begin{array}{l}\text { Chicken wire near vege- } \\
\text { tation }\end{array}$ \\
\hline 4 & 232 & Mesh screen \\
\hline 5 & 250 & $\begin{array}{l}\text { Chicken wire concealing } \\
\text { mortar stand }\end{array}$ \\
\hline 6 & 253 & $\begin{array}{l}\text { Sniper blind concealing } \\
\text { dummy with rocket } \\
\text { launcher }\end{array}$ \\
\hline 7 & 238 & Fencing in front of tree \\
\hline 8 & 68 & $\begin{array}{l}\text { Dummy in trench with } \\
\text { covered with fencing }\end{array}$ \\
\hline 9 & 45 & $\begin{array}{l}\text { Tent concealing rifle on } \\
\text { tripod }\end{array}$ \\
\hline
\end{tabular}

Table 2: Target descriptions and their ranges from the scanners

\subsection{Range ambiguity}

Targets 1 and 2 were chosen to study the range ambiguity of the scanners more in-depth. Target 1 was covered by a camouflage netting and had a wooden vertical support stand in front of a white painted background board. The front, central portion of Target 1 


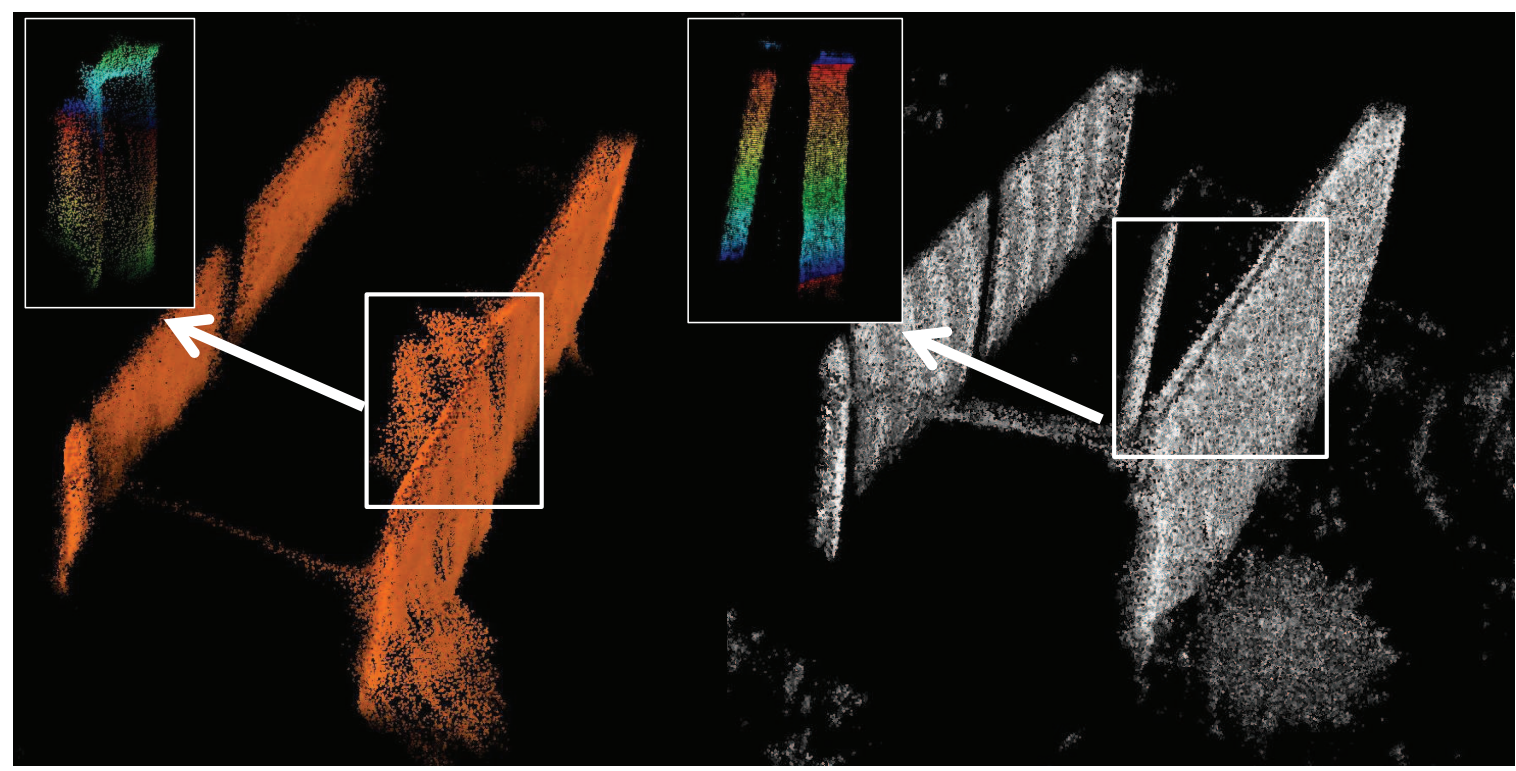

Figure 2: (Top) Point clouds of Target 1 from (left) VZ-400 and (right) HRS-3D. Insets show chosen point cloud subsets for further analysis.

was isolated for the following analysis of both point clouds as seen in the insets of Fig 2. The histograms of Target 1's isolated range returns for the VZ-400 and HRS-3D is seen at top and bottom of Figure 3, respectively. There is a rather stark contrast between the two range return histograms. As can be seen in the top plot of the VZ-400 range return histogram, there are two range return peaks at approximately $220.6 \mathrm{~m}$ and $220.75 \mathrm{~m}$, and a smaller, less distinct peak at approximately $220.95 \mathrm{~m}$. The first two peaks are due to the netting on the left and right side of the vertical support. However, the netting directly in front of the support does not appear as a return in the data. Instead, the returns appear ambiguous as many are registered between the netting and support stand. The support stand itself is the ill-defined peak at $220.95 \mathrm{~m}$.

Meanwhile, the HRS-3D returns rendered significantly, betterdefined surfaces for the netting and vertical support. The netting surface is the peak at $221 \mathrm{~m}$ in the bottom histogram of Figure 3 . The vertical support is the sharp peak at $221.5 \mathrm{~m}$. There are only a few stray points which appear between the netting and vertical support surfaces in the HRS-3D data. These stray points are mainly located between ranges of $221.15 \mathrm{~m}$ and $221.35 \mathrm{~m}$.

Two sections of Target 2 were chosen to compare the range ambiguities of the two scanners. The isolated sections are outlined in white boxes in 4 and the insets show the point cloud subsets. The first section included the double-layered mesh, vertical support, and backboard of Target 2. The range return histograms for the first section are shown in Figure 5 for the VZ-400 (top) and HRS-3D (bottom). Interestingly, the VZ-400 histogram shows 4 distinct peaks. The first peak at $184.5 \mathrm{~m}$ is due to the doublelayered mesh surface. However, the double-layered mesh is not resolved by the VZ-400 into two separate surfaces. The second and third peaks are located at $185 \mathrm{~m}$ and $185.15 \mathrm{~m}$, respectively. The double peaks here are due to the range "pulling" effect induced by the mesh. This effect is due (in part) to: 1) the inability of the linear mode system to resolve objects outside of its pulse bandwidth and 2) movement of the mesh material during the scan event. The upper portion of the vertical support has an unabated line-of-sight to the scanner while the lower portion has the double layer mesh intervening the scanner's line-of-sight to the vertical support. The mesh interference shifted the range return of the
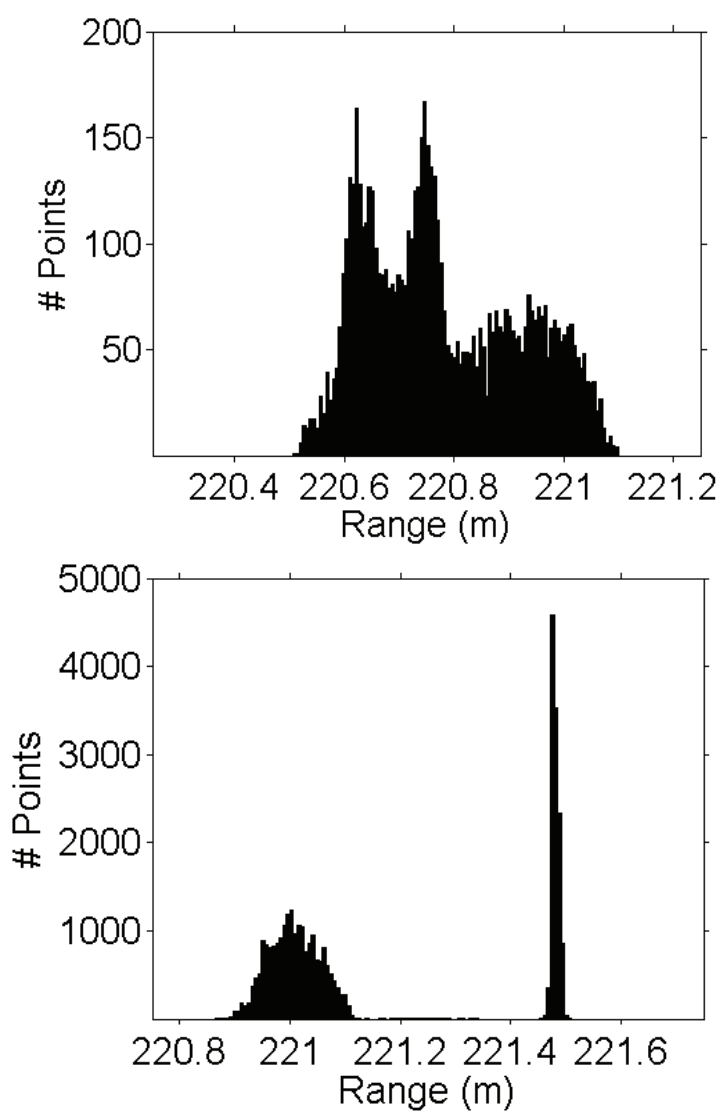

Figure 3: Range return histograms of Target 1 area for (Top) VZ400 and (Bottom) HRS-3D

lower portion of the vertical support approximately $15 \mathrm{~cm}$ closer. The backboard return is located at approximately $185.7 \mathrm{~m}$. There is also a considerable "filling-in" of returns between the mesh and vertical support in the VZ-400 data as can be seen between 184.5 and $185 \mathrm{~m}$. 


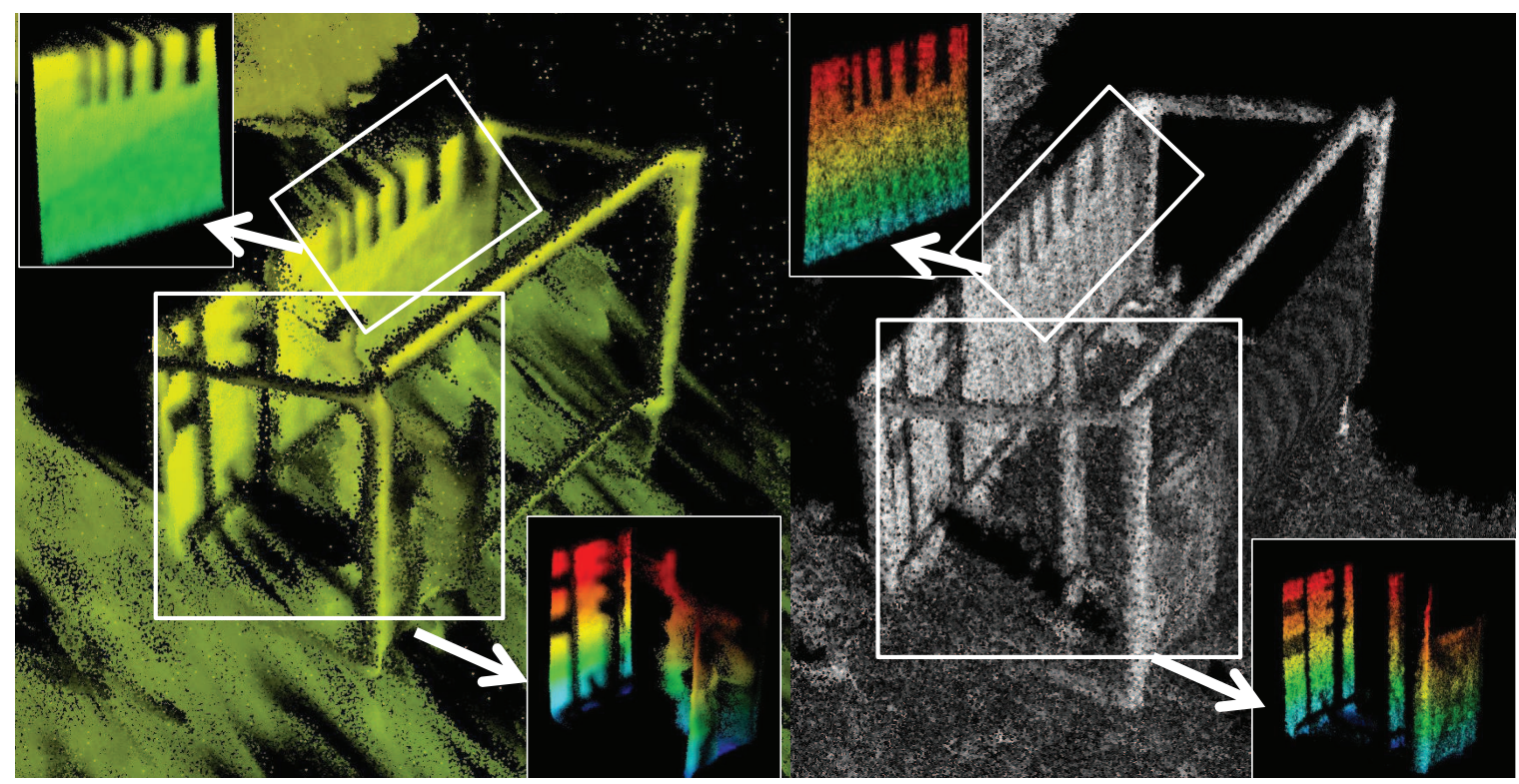

Figure 4: (Top) Point clouds of Target 2 from (left) VZ-400 and (right) HRS-3D. Insets show chosen point cloud subsets for further analysis.
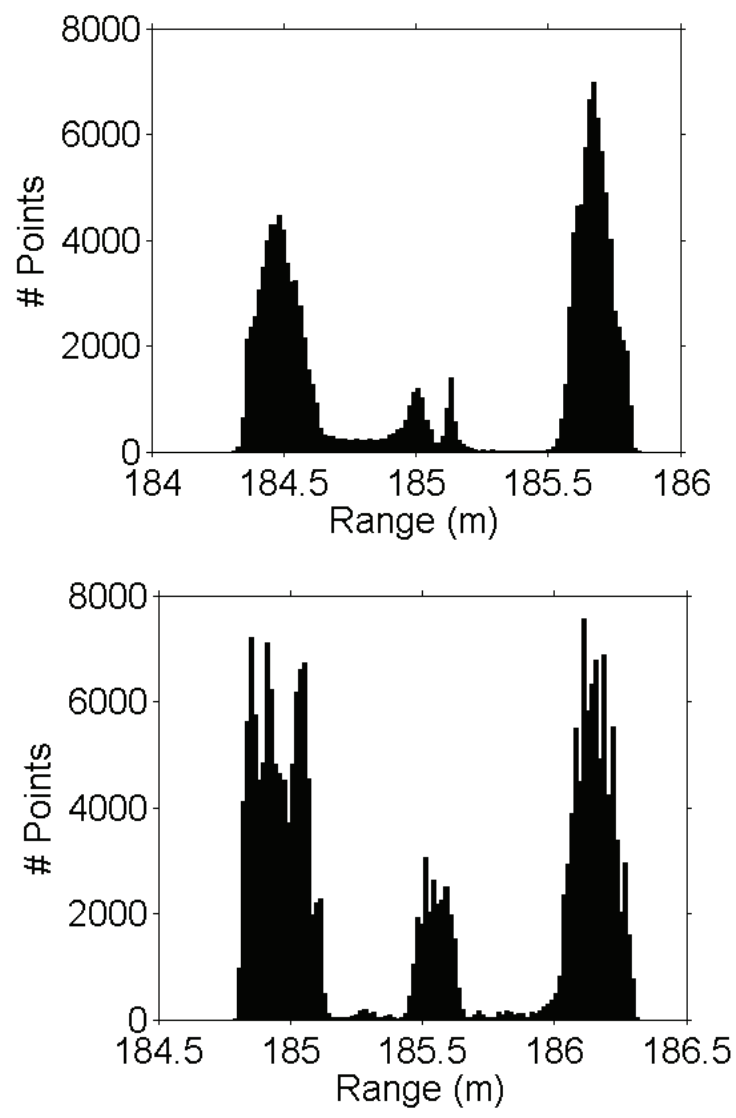

Figure 5: Range return histograms of Target 2 mesh, vertical support, and backboard for (Top) VZ-400 and (Bottom) HRS-3D

The HRS-3D data shows three distinct range return peaks around $185 \mathrm{~m}$. Two of these are due to the double-layered mesh (a separation of only a few $\mathrm{cm}$ ) while the third peak is due to the horizontal frame at the bottom of Target 2 . The central vertical support at $185.55 \mathrm{~m}$ and the backboard at $186.2 \mathrm{~m}$ are both clearly defined in the range returns. There are a few spurious returns in the HRS3D data located at $185.3 \mathrm{~m}$ range. The fill-in observed between the vertical support and the backboard at ranges of $185.7 \mathrm{~m}$ to $186 \mathrm{~m}$ is due to low-lying vegetation.
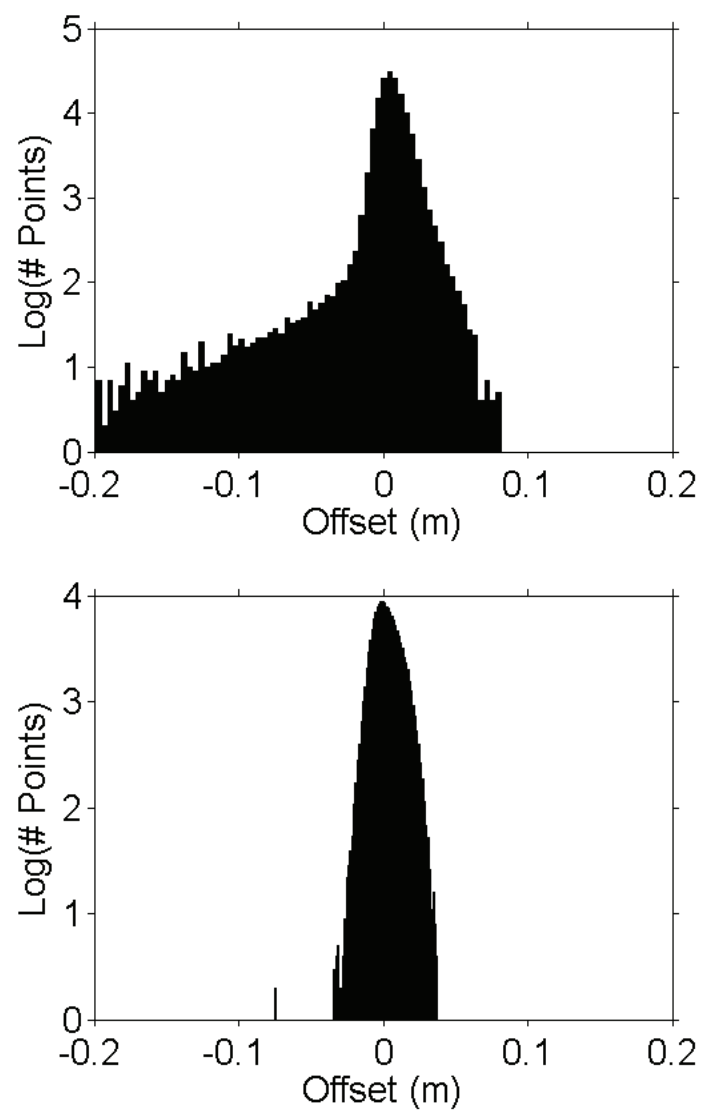

Figure 6: Log histograms of offset from Target 2 backboard for (Top) VZ-400 and (Bottom) HRS-3D 


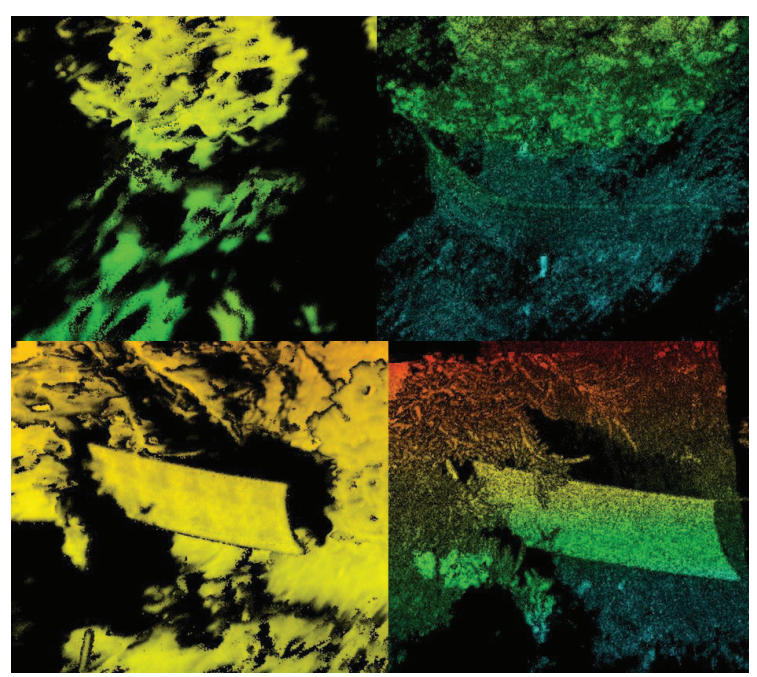

Figure 7: Point clouds of Targets 3 (top) and 4 (bottom), VZ-400 and HRS-3D point clouds are on the left and right sides, respectively.

The second section isolated from Target 2 is the backboard containing the vertical black resolution test lines as can be seen in Figure 4 top-left insets. For each of the sub-setted point clouds, a vertical plane was calculated to act as the geometrical representation of the backboard. The distance between the backboard plane and each point was then calculated. This distance is the offset between the 3-D data point and the vertical plane. Logarithm histograms of the distance offsets are shown in Figure 6 for the VZ-400 (top) and HRS-3D (bottom). Negative and positive distance offset values represent points behind and in front of the backboard from the scanners' viewpoints, respectively. The VZ400 histogram shows a distinct negative offset shoulder. This is due to range ambiguities between the top of the backboard and a tree limb located approximately $0.5 \mathrm{~m}$ behind Target 2 . The positive distance offset shoulder shows that the VZ-400 registered returns out to $8 \mathrm{~cm}$ in front of the backboard. Meanwhile, the HRS-3D histogram shows that there is no such range ambiguity between the backboard and the tree behind Target 2. Additionally, the distance offsets are confined to $\pm 5 \mathrm{~cm}$ from the backboard plane.

\subsection{Obscurant penetration}

In this section, the obscurant penetration performance of the two scanners is compared. Some of the differences have already been demonstrated from Targets 1 and 2 in the previous section. Figures 7 and 8 show the remainder of the targets' point clouds. The VZ-400 point clouds are on the left side while the HRS-3D point clouds are on the right side. In Target 3 (Figure 7 top), it can be seen that the VZ-400 point cloud (left) is far sparser than the HRS-3D point cloud (right) behind the chicken wire fencing. Low-lying vegetation can be seen in the HRS-3D data but is unrecognizable in the VZ-400 data. In Target 4 (Figure 7 bottom), it can be seen that both scanners could not easily achieve consistent penetration through the mesh screen. However, the HRS-3D was able to register approximately $10 \%$ of the total returns had the intervening screen not been present. The VZ-400 did not register any returns from behind the mesh screen. In Target 5 (Figure 8a), the VZ-400 did not register any returns from the mortar stand behind the chicken wire (see inset for top-down view. The HRS-3D was able to clearly resolve the mortar stand's two legs and barrel. In Target 6 (Figure 8b), the VZ-400 was unable to register the dummy with the rocket launcher behind the sniper blind. Inter- estingly, however, the intensity image of the dummy's head and shoulders can be seen overlaid on the sniper blind. The HRS-3D was able to easily resolve the dummy's head, torso, and limbs and was able to resolve the barrel of the rocket launcher. Both scanners were able to easily penetrate the fencing of Target 7 (Figure 8c). However, the HRS-3D was slightly more successful than the VZ-400 at registering points on the actual fenceline. In Target 8 (Figure 8d), the VZ-400 was unable to geometrically resolve the dummy in the ditch under the fencing. However, similar to Target 6 , the dummy's head appears as higher intensity points mapped onto the surface of the obscuring fenceline. The HRS-3D is able to geometrically resolve the dummy's head, torso, and arms. Finally, in Target 9 (Figure 8e), both scanners are able to penetrate the tent lining to resolve the propped-up rifle inside. The HRS$3 \mathrm{D}$ resolves features such as the scope and the shoulder strap. The VZ-400 does a better job at registering the intensity changes of the tent door flap.

\subsection{Maximum range}

Several measurements were made in this study to assess the maximum range capabilities of the two scanners. The VZ-400 and HRS-3D were both capable of registering returns from highly reflective surfaces at a range of $900 \mathrm{~m}$. The surfaces were primarily vegetation and thus possessed exceptional reflectance (0.7) at $1.55 \mu \mathrm{m}$. The HRS-3D registered approximately twice as many returns than the VZ-400 at these long ranges.

\section{ANALYSIS}

From the standpoint of tactical, military targeting and topographic rendition, it is clear from this study that the FMCW technology vastly improves range ambiguity and obscurant penetration of terrestrial laser scanners. The returns from closely spaced surfaces (down to approximately $10 \mathrm{~cm}$ in separation) are capable of being range-resolved by the HRS-3D. In contrast, the VZ-400 begins to experience range ambiguity problems when surface separations are at approximately $0.5 \mathrm{~m}$ or less. This is especially true for closely spaced surfaces at larger ranges, as can be seen in Targets $1,2,5$, and 6 . The VZ-400 tends to do a better job at range resolution and obscurant penetration when the targets are closer in range, as in Target 9. Interestingly, the VZ-400 scanner recorded higher intensity values from the concealed objects mapped onto the surfaces of the obscurants as in Targets 6 and 8. It may be the case that the concealed object's Gaussian return shoulder is having an additive effect to the obscurant's surface return. Yet, the concealed object's Gaussian peak is not strong enough to be registered as its own 3-D return by the sensor. It was also observed that the HRS-3D displayed remarkable vegetation feature resolution at larger ranges. Tactically, this is particularly important in foliage penetration(Massaro et al., 2012) and the rendition of camouflage. It is believed that FMCW-LADAR systems will be more adept at highly accurate vegetation mapping(Pirotti et al., 2013).

\section{CONCLUSION}

While the FMCW system certainly outperformed the conventional, pulsed system in terms of range resolution, obscurant penetration, and maximum range, there are some drawbacks to the FMCW system. It currently takes about six times as long for a 3-D scene to be acquired with the FMCW system. The FMCW scene acquisition time can likely be decreased however with improved FPGA boards and optimized scanning techniques. The size, weight, and power requirements of the FMCW system are also significantly 


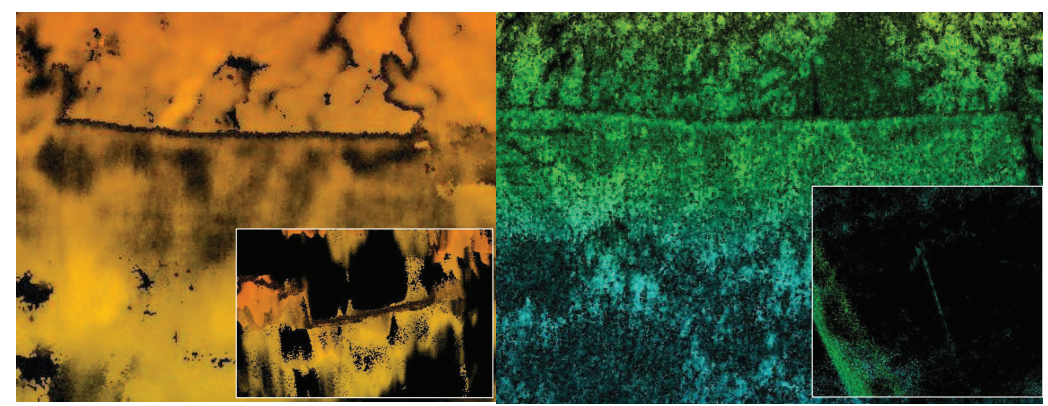

(a) Target 5 (insets are top-down views)

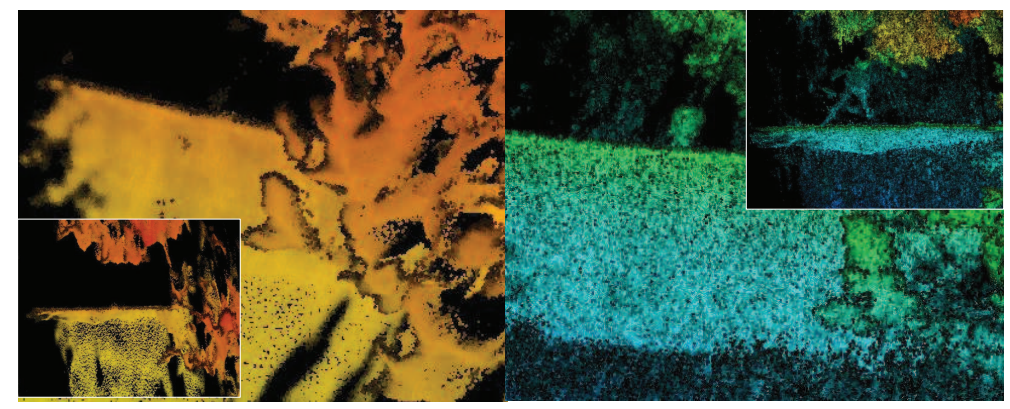

(b) Target 6 (insets are top-down views)

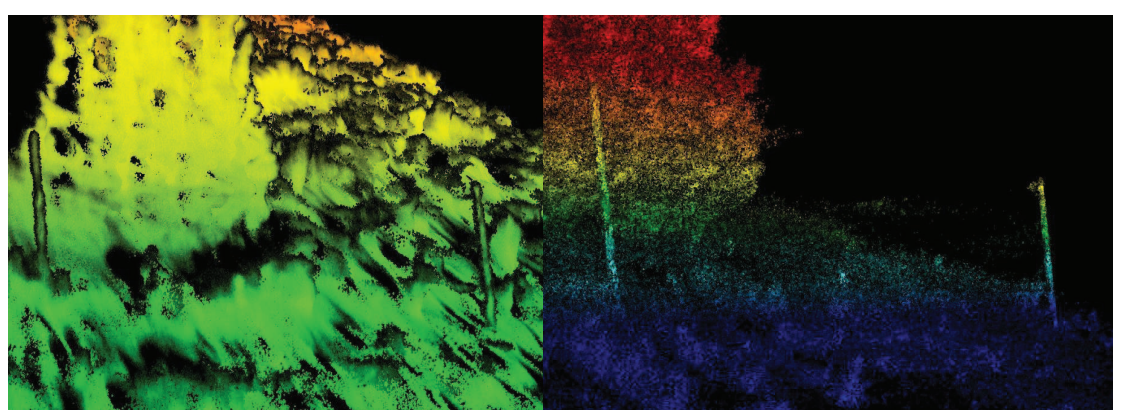

(c) Target 7

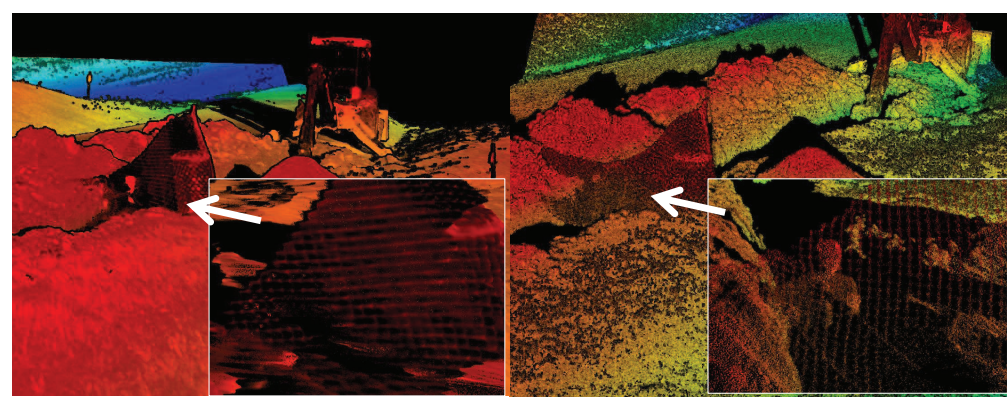

(d) Target 8 (insets show close-up of dummy location)

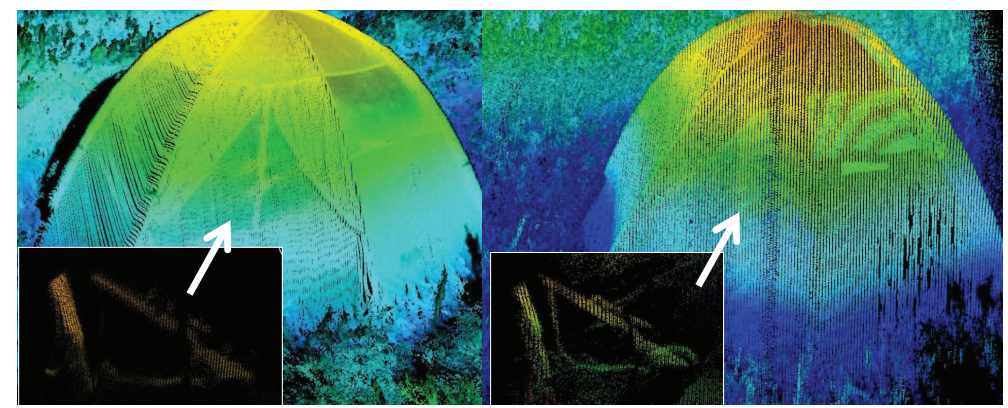

(e) Target 9 (insets show points from tent interior)

Figure 8: Point clouds of Targets 5 through 9 from top to bottom. VZ-400 and HRS-3D point clouds are on the left and right sides, respectively. 
greater than the slimmer conventional system. However, this can be remedied by eventual inclusion of the processing unit into the scanner head.

As compared to heterodyne laser radar systems nearly 30 years ago(Keyes, 1986), FMCW-LADAR seems to have overcome many technological hurdles. In all, it seems clear that the future of terrestrial laser scanning, and possibly air- and space-borne LADAR, lies in FMCW systems. In addition to the superiority of their range resolution and obscurant penetration, FMCW systems can provide Doppler velocities and theoretically achieve greater maximum range given the same power input.

\section{ACKNOWLEDGEMENTS}

The authors are grateful to Dr. Randy Reibel, Jim Curry, Trent Berg, and the rest of the team from Bridger Photonics for their willingness and assistance in collecting and processing the test data.

\section{REFERENCES}

Keyes, R. J., 1986. Heterodyne and nonheterodyne laser transceivers. Review of Scientific Instruments 57, pp. 519-528.

Massaro, R., Zinnert, J., Anderson, J., Edwards, J., Crawford, E. and Young, D., 2012. Lidar flecks: modeling the influence of canopy type on tactical foliage penetration by airborne, active sensor platforms. In: Airborne Intelligence, Surveillance, Reconnaissance (ISR) Systems and Applications IX, Proc. SPIE, Vol. 8360 , p. 836008 .

Pirotti, F., Guarnieri, A. and Vettore, A., 2013. Ground filtering and vegetation mapping using multi-return terrestrial laser scanning. ISPRS Journal of Photogrammetry and Remote Sensing 76, pp. 56-63.

Reibel, R. R., Greenfield, N. J., Berg, T. J., Kaylor, B. M. and Roos, P. A., 2010. Ultra-compact ladar systems for next generation space missions. In: Conference on Small Satellites, Vol. SSC10-I-9, pp. 1-11.

Reibel, R. R., Roos, P. A., Kaylor, B. M., Berg, T. J. and Curry, J. R., 2014. Imaging through obscurants with a heterodyne detection-based ladar system. In: J. J. G (ed.), Degraded Visual Environments: Enhanced, Synthetic, and External Vision Solutions.

Shan, J. and Toth, C. K., 2009. Topographic Laser Ranging and Scanning: Principles and Processing. CRC Press. 